\title{
Cargo-carrier interactions significantly contribute to micellar conformation and biodistribution
}

\author{
Adrian T Press ${ }^{1,2}$, Anuradha Ramoji ${ }^{2,3}$, Moritz vd Lühe ${ }^{4,5}$, Alexandra C Rinkenauer ${ }^{4,5}$, Jessica Hoff ${ }^{1}$, \\ Marianne Butans ${ }^{1,2}$, Carsten Rössel ${ }^{4,5}$, Christian Pietsch ${ }^{4,5}$, Ute Neugebauer ${ }^{2,3,5}$, Felix H Schacher ${ }^{4,5}$ \\ and Michael Bauer ${ }^{1,2,5}$
}

\begin{abstract}
Strategies to deliver drugs using nanocarriers, which are passively or actively targeted to their alleged site of action might favorably affect benefit-risk profiles of novel therapeutics. Here we tested the hypothesis whether the physico-chemical properties of the cargo as well as the actual conditions during encapsulation interfere during formulation of nanoparticular cargo-carrier systems. On the basis of previous work, a versatile class of nanocarriers is polyether-based ABC triblock terpolymer micelles with diameters below $50 \mathrm{~nm}$. Their tunable chemistry and size allows to systematically vary important parameters. We demonstrate in vivo differences in pharmacokinetics and biodistribution not only dependent on micellar net charge but also on the properties of encapsulated (model) drugs and their localization within the micelles. On the basis of in vitro and in vivo evidence we propose that depending on drug cargo and encapsulation conditions micelles with homogeneous or heterogeneous corona structure are formed, contributing to an altered pharmacokinetic profile as differences in cargo location occur. Thus, these interactions have to be considered when a carrier system is selected to achieve optimal delivery to a given tissue.
\end{abstract} NPG Asia Materials (2017) 9, e444; doi:10.1038/am.2017.161; published online 27 October 2017

\section{INTRODUCTION}

Strategies to target drugs passively or actively to specific cells or tissues may allow interference with central signaling events in health and disease. For example, effector kinases are promising targets for therapy in diverse disease entities ranging from cancer to organ dysfunction in critical care. ${ }^{1,2}$ Interventions into kinase function (for example, PI3K) might be limited by deleterious off-target effects, for example, on the immune system. A broad spectrum of approaches, including viruses and non-viral vectors has been applied for delivery to specific cells, but these strategies are still far from satisfactory. Besides liposomes, polymer-based nanocarriers are the largest class of non-viral vectors. Their pharmacology and toxicology were shown to depend on size, composition, surface charge (zeta potential) and shape; these key factors seemingly determine nanoparticle uptake, immune cell recognition and circulation time. ${ }^{3-7}$ Often carriers with cationic net charge interact strongly with and are capable of penetrating the cellular membrane, leading to high delivery efficiencies but this is often accompanied by impaired cell viability and clearance by the reticuloendothelial system. ${ }^{8,9}$

An interesting class of nanocarriers is block copolymer micelles with diameters below $50 \mathrm{~nm}$ as these are reported to deeply penetrate tumor tissue in spite of elevated interstitial pressure. ${ }^{10}$ In addition,
PEGylation generally facilitates long circulation times while, ideally, a surface-tag might induce specific interaction of the particle with the targeted cells or tissues. ${ }^{11}$ Unfortunately, pharmacokinetic studies of the generated nanoparticles often tell a different story: effects such as the accelerated blood clearance are just one obstacle often rendering even PEGylation approaches unsuccessful. ${ }^{12,13}$

The self-assembly of block copolymers in selective solvents is an elegant way to create nanocarriers with defined size, charge and surface chemistry. ${ }^{14}$ In the case of $\mathrm{ABC}$ triblock terpolymers, even further design criteria can be accomplished and well-defined structures of different morphology featuring core, shell and corona layers can be fabricated and used as potential drug delivery vehicles. ${ }^{15-18}$ We have recently shown that multicompartment micelles from polybutadiene-block-poly(methacrylic acid)-block-poly( $N, N$-dimethylaminoethyl methacrylate) (BMAAD) undergo structural rearrangements within a relatively narrow $\mathrm{pH}$ regime (5-7) and thus are promising carriers for the transfection of pDNA into both adherent cells and cells in suspension. ${ }^{19}$ To enhance flexibility and improve control over composition, charge and charge density within coreshell-corona micelles, we developed a platform approach based on co-assembly of polyether-based ABC triblock terpolymers. Here poly (ethylene oxide)-block-poly(allyl glycidyl ether)-block-poly(tert-butyl

\footnotetext{
${ }^{1}$ Department of Anesthesiology and Intensive Care Medicine, Jena University Hospital, Jena, Germany; ${ }^{2}$ Center for Sepsis Control and Care, Jena University Hospital, Jena, Germany; ${ }^{3}$ Leibniz-Institute for Photonic Technology, Jena, Germany; ${ }^{4}$ Institute of Organic Chemistry and Macromolecular Chemistry, Friedrich-Schiller-University Jena, Jena, Germany and ${ }^{5}$ Jena Center for Soft Matter (JCSM), Friedrich-Schiller-University Jena, Jena, Germany

Correspondence: Professor Dr M Bauer, Department of Anesthesiology and Intensive Care Medicine, Jena University Hospital, Am Klinikum 1, Jena D-07747, Germany. E-mail: michael.bauer@med.uni-jena.de

or Professor Dr FH Schacher, Institute of Organic Chemistry and Macromolecular Chemistry, Friedrich-Schiller-University Jena, Lessingstraße 8, D-07743 Jena, Germany. E-mail: felix.schacher@uni-jena.de

Received 16 May 2017; accepted 26 June 2017
} 
glycidyl ether) (PEO- $b$-PAGE- $b$-PtBGE) were prepared using sequential living anionic ring-opening polymerization. ${ }^{20}$ The presence of side-chain functionalities in the PAGE block allowed the selective subsequent introduction of $-\mathrm{NH}_{2},-\mathrm{COOH}$ or galactose moieties using thiol-ene chemistry. Moreover, self-assembly resulted in micelles with identical core- and corona-forming segments, but different chemistry in the shell. Co-assembly of materials with different functionalities in the B segment enabled control over important micellar characteristics, influencing cytotoxicity and uptake in vitro. ${ }^{21} \mathrm{We}$ also found evidence that uptake mechanism depends on shell composition, motivating us to further investigate pharmacokinetics of these nanostructures. In our opinion, an often neglected aspect in the context of drug delivery are (favorable or unfavorable) interactions between cargo and carrier during encapsulation-this might drastically influence the overall amount of guest molecules encapsulated as well as their specific location and, with that, accessibility toward release. The above-described triblock terpolymers based on polyethers might thus serve as a versatile platform for purposeful variation of carrier characteristics and polarity/charge density during encapsulation of suitable model cargo.

We herein demonstrate in both in vitro as well as in vivo studies time-dependent uptake, uptake mechanism and elimination of coreshell-corona micelles with different shell composition. Thereby, PEO$b$-PAGE $\mathrm{NH}_{2}-b$-PtBGE forms micelles with cationically charged groups present in the shell (ENT), while PEO- $b$-PAGE $\mathrm{COOH}^{-} b$-PtBGE features anionic charges (ECT) and, finally, mixtures of ENT/ECT exhibit net charge depending on the mixing ratio (EMT). To trace such micelles by multiple fluorescence techniques, we incorporate either Nile red (NR) or V19-05048 (V19) by encapsulation during micelle formation in water as selective solvent and applied Raman imaging as a label-free method to study their composition. ${ }^{22-24} \mathrm{We}$ are able to show that depending on the preparation conditions, the location of V19 as a surrogate drug within the micelles can be influenced and that this has significant effects on the pharmacokinetic profile of these micelles. Further, depending on its location within the nanostructures, the release of V19 in the intact liver can be demonstrated by characteristic accumulation within mitochondria.

\section{MATERIALS AND METHODS}

\section{Preparation of triblock terpolymer micelles}

Tetrahydrofuran (THF) and the dyes NR and 1,6-diphenyl-1,3,5-hexatriene (DPH) were purchased from Sigma Aldrich (Taufkirchen, Germany) and used as received. The dye V19-05048 was purchased from Dyomics GmbH (Jena, Germany). PEO-b-PAGE- $b$-PtBGE was synthesized and modified using thiol-ene chemistry as described previously. ${ }^{21}$ The micelles (ECT and ENT) were prepared according to literature ${ }^{21}$ as follows: in total, $50 \mathrm{mg}$ of the corresponding triblock terpolymer was dissolved in $2.5 \mathrm{ml}$ of THF, and $2.0 \mathrm{mg}$ of NR or V19 was added to the solution. In a next step $5.0 \mathrm{ml}$ of deionized water (or aqueous solution of $\mathrm{pH} 4$ ) was very slowly added with a syringe under stirring. Afterwards, the THF was allowed to evaporate by stirring overnight. Non-encapsulated dye was filtered off by a $0.45 \mu \mathrm{m}$ nylon syringe filter. After filtration, a clear-colored solution could be obtained. The solutions were then diluted with deionized water to a concentration of $1 \mathrm{gl}^{-1}$.

\section{Determination of micellar stability for ECT}

The micellar stability of ECT was determined through encapsulation of DPH. Different volumes of a stock solution of $0.5 \mathrm{mg} \mathrm{ml}^{-1}$ ECT in THF were mixed with $800 \mu \mathrm{l}$ of a $0.4 \mathrm{~mm}$ DPH solution in THF. THF was added to obtain $1 \mathrm{ml}$ total volume for every sample. Subsequently, $1 \mathrm{ml}$ of micropure water was added dropwise and the samples were stirred open at room temperature for $24 \mathrm{~h}$ to evaporate THF. Afterwards, $1 \mathrm{ml}$ of $\mathrm{KH}$ buffer was added and ultraviolet/visible spectra were recorded before and after filtration through a
$450 \mu \mathrm{m}$ nylon syringe filter on a Cary 60 spectrometer (Agilent Technologies, Santa Clara, CA, USA). In addition, $6.6 \mathrm{mg}$ ECT and $1 \mathrm{mg}$ NR were dissolved in $1 \mathrm{ml}$ THF and $2 \mathrm{ml} \mathrm{KH}$ buffer was added dropwise. After stirring openly overnight to evaporate THF, the resulting micellar solution was filtered through a $0.45 \mu \mathrm{m}$ nylon syringe filter to remove non-encapsulated NR and the concentration was adjusted using $\mathrm{KH}$ buffer. Fluorescence spectra in the range of $525-750 \mathrm{~nm}$ were acquired on a FP-6500 Spectrofluorometer (Jasco, Pfungstadt, Germany) with an excitation wavelength of $520 \mathrm{~nm}$ at $25^{\circ} \mathrm{C}$.

\section{Binary co-micelles}

The binary co-micelles (EMT) were prepared with a 3.5:1 mixture of $\mathrm{PEO}_{42}-b$ $\left(\mathrm{PAGE}_{8, \mathrm{NH} 2}-c o-\mathrm{PAGE}_{7}\right)-b-\mathrm{PtBGE}_{12}$ (ENT) and $\mathrm{PEO}_{42}-b-\mathrm{PAGE}_{15}, \mathrm{COOH}^{-} b-$ $\mathrm{PtBGE}_{12}$ (ECT). Because of the different degrees of functionalization, a 3.5:1 ratio regarding their masses (mass $\%$ of the corresponding repeating unit) was used. A unit of $20.5 \mathrm{mg}$ (correspond to $5.46 \mathrm{mg} \mathrm{NH} \mathrm{N}_{2}$ units) of $\mathrm{PEO}_{42}-b$ $\left(\mathrm{PAGE}_{8, \mathrm{NH} 2}-\mathrm{co}-\mathrm{PAGE}_{7}\right.$ ) $-b-\mathrm{PtBGE}_{12}$ and $3.2 \mathrm{mg}$ (correspond to $1.56 \mathrm{mg} \mathrm{COOH}$ units) of $\mathrm{PEO}_{42}-b$ - $\mathrm{PAGE}_{15}, \mathrm{COOH}^{-} b-\mathrm{PtBGE}_{12}$ were dissolved in $1.0 \mathrm{ml}$ THF and $0.8 \mathrm{mg}$ of NR or V19 was added to the solution. In a next step $2.0 \mathrm{ml}$ of deionized water was very slowly added via syringe under stirring. Afterwards, the THF was allowed to evaporate by stirring overnight. Non-encapsulated dye was filtered off by a $0.45 \mu \mathrm{m}$ nylon syringe filter. After filtration, a clear-colored solution could be obtained. The solutions were then diluted with deionized water (or aqueous solution of $\mathrm{pH} 4$ ) to a concentration of $1 \mathrm{gl}^{-1}$.

\section{Dynamic light scattering}

Dynamic light scattering (DLS) was performed at a scattering angle of $90^{\circ}$ on an ALV CGS-3 instrument (Langen, Germany) and a He-Ne laser operating at a wavelength of excitation $=633 \mathrm{~nm}$ at $25^{\circ} \mathrm{C}$. The CONTIN algorithm was applied to analyze the obtained correlation functions. Apparent hydrodynamic radii were calculated according to the Stokes-Einstein equation. All CONTIN plots are number-weighted.

\section{Electrophoretic light scattering}

Electrophoretic light scattering was applied to measure the electrokinetic potential using a Zetasizer Nano ZS (Malvern Instruments, Herrenberg, Germany) by applying laser Doppler velocimetry. For each measurement, 30 runs were carried out using the slow-field reversal and fast-field reversal modes at $150 \mathrm{~V}$. Each experiment was performed in triplicate at $25^{\circ} \mathrm{C}$. The zeta potential $(\zeta)$ was calculated from the electrophoretic mobility as described before for these micelles. ${ }^{21}$

\section{Correction factor}

A correction factor was measured and calculated as previously described ${ }^{25}$ using a fluorescent plate reader (Tecan Infinite Pro200, Crailsheim, Germany) to compare between different micelles loaded with the same probe.

\section{Fluorescence spectroscopy}

Spectra were measured on a fluorescent plate reader (Tecan Infinite Pro200). Micelles were measured in deionized water and V19 as well as NR in pure ethanol, as their hydrophobicity did not allow the measurement in aqueous environment. For excitation scans the emission wavelength was set to $680 \pm 20 \mathrm{~nm}$ for NR and $620 \pm 20 \mathrm{~nm}$ for V19. The emission scans were performed while exciting the dyes at $530 \pm 10$ or $440 \pm 20 \mathrm{~nm}$, respectively.

\section{Cell culture}

HEK293 cells (CLR-1573, American Type Culture Collection, Manassas, VA, USA) were maintained in RPMI 1640 and HepG2 (DSMZ, Braunschweig, Germany) were cultured in Dulbecco's modified Eagle's medium/Ham's F-12. The media were supplemented with $10 \%$ fetal calf serum, $100 \mathrm{IU}$ of streptomycin and $100 \mathrm{IU}$ of penicillin, and in the case of the HEK293 cells 2 mM L-glutamine were added.

\section{Raman imaging}

HepG2 were cultured on $\mathrm{CaF}_{2}$ slides for $48 \mathrm{~h}$. Cells were then incubated for 30 min with ECT or ENT without payload and fixed subsequently with $0.5 \%$ 
formaldehyde (Carl Roth, Karlsruhe, Germany) in phosphate-buffered saline (Biochrom, Berlin, Germany) for $10 \mathrm{~min}$. Afterwards the samples were washed twice and stored at $4{ }^{\circ} \mathrm{C}$ in phosphate-buffered saline for imaging. Raman spectra of the cells were measured using an upright micro-Raman system (CRM 300, WITec GmbH, Ulm, Germany) equipped with a $300 \mathrm{~g} \mathrm{~mm}^{-1}$ grating $\left(0.4 \mu \mathrm{M}\right.$ spatial resolution and $7 \mathrm{~cm}^{-1}$ spectral resolution) and a Deep Depletion charge-coupled devise camera (DU401 BR-DD, ANDOR, Belfast, UK $-1024 \times 127$ pixels) cooled down to $-60{ }^{\circ} \mathrm{C}$. The cells were excited with excitation wavelength of $785 \mathrm{~nm}$ diode laser (laser power on object plane $75 \mathrm{~mW}$ ) and the back-scattered Raman signals were collected using $\times 60$ objective (numerical aperture; NA 1.0). With the Raman setup used in the present work $(785 \mathrm{~nm}$ excitation wavelength and $\times 60 / \mathrm{NA} 1.0)$ the limit of diffraction can be calculated using Abbe's formula: $\lambda / 2 \mathrm{NA}=785 / 2=392.5 \mathrm{~nm}$. To justify the Nyquist criteria we should sample with a step size of $\lambda / 4 \mathrm{NA}=196.25 \mathrm{~nm}$. This was done by using a step size of $200 \mathrm{~nm}$ to generate the presented images. The cellular region was mapped by collecting point-bypoint spectra from the entire region with a step size of $0.2 \mu \mathrm{M}$ and acquisition time of $2 \mathrm{~s}$ per spectrum.

Raman image was then analyzed using MATLAB (8.0.0.783 (R2012b)) with in-house-built script NFINDR for spectral unmixing algorithm (Mathworks, Natick, MA, USA). ${ }^{26}$ Raman spectra were pre-processed, which included steps such as cosmic spike removal, principal component analysis based noise reduction, getting rid of non-cellular background using $k$-means cluster analysis, removing spectral background and normalization. Finally, the fingerprint region $\left(600-1800 \mathrm{~cm}^{-1}\right)$ and the $\mathrm{CH}$ stretching region (2800-3000 $\left.\mathrm{cm}^{-1}\right)$ were used. ECT and ENT signal intensities in treated and control HepG2 cells were local background-subtracted (mean intensity of $50 \mathrm{px}$ radius), smoothed (median filter, $1 \mathrm{px}$ ) and false-color coded (16 color heat map) using Image $1.50 \mathrm{e}$ (National Institute of Health, Bethesda, MD, USA). The Raman spectra of different micelles with and without payloads and the respective polymers were collected using $\times 100$ objective (NA 0.75) as follows: drop of the samples was taken on $\mathrm{CaF}_{2}$ slides and a region of $10 \times 10 \mu \mathrm{m}$ was scanned with a spectral acquisition time of $10 \mathrm{~s}$. The Raman spectra were pre-processed using GNU R programming platform with in-house-built script (Vienna, Austria). ${ }^{27}$ The pre-processing involved spectral background correction $^{28}$ and normalization. Each spectrum shown (Figures 2 and 4) is average of $\sim 400$ spectra. The Raman spectra of payloads were measured with $10 \mathrm{~s}$ of acquisition time. The spectra are pre-processed in similar way as Raman spectra of micelles and polymers.

\section{Live-cell imaging}

HEK293 or HepG2 cells were cultured in CellView Chamber slides (Greiner BioOne, Frickenhausen, Germany) under optimal conditions as described above. Directly before imaging were stained with $\mathrm{H} 33342$ as previously described. ${ }^{24}$ For co-staining experiments tetramethylrhodamine and V19 were added simultaneously with equimolar concentration $\left(200 \mathrm{nmoll}^{-1}\right)$ in full media. After 15 min cells were washed twice with Hanks' balanced salt solution and fresh media was added for imaging. Detection of tetramethylrhodamine and V19 was done as described before. ${ }^{24}$

In addition, cells were incubated for some experiments in fetal calf serumfree media containing $25 \mu \mathrm{m}$ Pitstop-2 (Abcam, Cambridge, UK) for $12 \mathrm{~min}$ as previously described. ${ }^{25}$ In brief, after washing the cells twice with Hanks' balanced salt solution, $480 \mu \mathrm{l}$ OptiMEM (Gibco, Thermo Scientific-both Langenselbold, Germany) or full-growth media was added. Cells were imaged in a humidified chamber at $37^{\circ} \mathrm{C}, 5 \% \mathrm{CO}_{2}$. ECT, ENT, EAT or EMT containing V19 or NR was added to the cells and imaged over 30 min. H33342 (Sigma Aldrich) was excited at $405 \mathrm{~nm}$; an emitted light between 410 and $480 \mathrm{~nm}$ was detected on a photomultiplier tube. V19 or NR was excited at $488 \mathrm{~nm}\left(\mathrm{Ar}^{+}\right.$laser$)$or $561 \mathrm{~nm}$ (diode-pumped solid-state laser), and emitted light of $500-580$ or $575-680 \mathrm{~nm}$ was detected on a GaAsP detector.

\section{Animals}

Animal studies were conducted in accordance with animal welfare legislation under pathogen-free conditions in the animal facility of the Jena University Hospital. During all procedures and imaging methods, animals remained under deep general anesthesia using isoflurane and pain reflexes were assessed to gauge the depth of anesthesia.

\section{In vivo confocal laser scanning microscopy}

For in vivo confocal laser scanning microscopy a tail-vein catheter (30 G) was placed in male FVB/NRj mice (22-30 g body weight). The liver was exposed and placed on a coverslip. Images were acquired using a LSM-780 (Zeiss AG, Jena, Germany) using air-corrected $\times 20$ plan-apochromatic (NA 1.15) or $\times 40$ plan-apochromatic objective (NA 0.95). $\mathrm{NADP}(\mathrm{H})$ autofluorescence of liver tissue was exploited to generate a tissue contrast as previously described. ${ }^{24}$ A unit of $200 \mu \mathrm{g}$ of different micelles were injected through the tail-vein catheter. In case of $\mathrm{ECT}\left(\mathrm{V} 19_{\mathrm{pH}}\right)$ the solution was adjusted to $\mathrm{pH} 7$ using technical-grade sodium hydroxide solution (Carl Roth) before injection. All solutions were filtered the day of usage though a $450 \mathrm{~nm}$ polyvinylidene fluoride syringe filter (Sigma Aldrich) to remove eventual precipitates. V19-05048 or NR was imaged as described above. Results are mean \pm s.e.m. of four biological replicates.

\section{Measurement of plasma disappearance rate and secretion}

Catheters were placed in the jugular vein, carotid artery, bladder and bile duct of male Wistar rats (230-280 g body weight). Micelles were administered intravenously ( $1 \mathrm{mg}$ per animal, solved in $1 \mathrm{ml}$ deionized water). ECT(V19 $\left.\mathrm{pH}_{4}\right)$ was neutralized and all micelles were filtered at the day of usage as described above. Bile fluid was collected every $10 \mathrm{~min}$ and $200 \mu \mathrm{l}$ arterial blood was taken at defined times into heparinized monovettes. Plasma was generated by centrifugation $(2000 \times g, 10 \mathrm{~min})$. Payload fluorescence was analyzed in the different body fluids using black 96-well plates (Greiner BioOne). Fluorescence was measured (V19: excitation: $485 \pm 29 \mathrm{~nm}$, emission: $560 \pm 20 \mathrm{~nm}$; or NR: excitation: $550 \pm 9 \mathrm{~nm}$, emission: $610 \pm 20 \mathrm{~nm}$ ) at a Tecan Infinite 200Pro. Standard curves for different micelles in the corresponding body fluid were used for quantification intensities. Results are mean \pm s.e.m. of three biological replicates.

\section{Cryogenic transmission electron microscopy}

Cryogenic transmission electron microscopy measurements were performed on a FEI Tecnai $G^{2} 20$ cryogenic-transmission electron microscope (Jena Center for Soft Matter). Acceleration voltages were set to $120 \mathrm{kV}$. Samples were prepared on Quantifoil (Jena, Germany) grids (3.5/1) after cleaning by argon plasma treatment for $120 \mathrm{~s}$. A volume of $9.5 \mu \mathrm{l}$ of the solutions were blotted by using a Vitrobot Mark IV (FEI, Eindhoven, Netherlands). Samples were plunge-frozen in liquid ethane and stored under nitrogen before being transferred to the microscope utilizing a Gatan transfer stage. Transmission electron microscopy images were acquired with a $200 \mathrm{kV}$ FEI Tecnai $\mathrm{G}^{2} 20$ equipped with a $4 k \times 4 k$ Eagle HS charge-coupled devise and a $1 k \times 1 k$ Olympus MegaView camera (Tokyo, Japan).

\section{RESULTS}

\section{Core-shell-corona micelles and co-micelles}

The triblock terpolymer PEO- $b$-PAGE- $b$-P $t$ BGE was synthesized using anionic ring-opening polymerization $\left(M_{\mathrm{n}}=5.100 \mathrm{~g} \mathrm{~mol}^{-1}\right.$, degree of polymerization of 42 (PEO), 15 (PAGE) and 12 (PtBGE), $Đ=1.05$ ). Subsequent modification was carried out using mercaptopropionic acid (PEO- $b$-PAGE $\mathrm{COOH}^{-} b$-PtBGE, quantitative functionalization of PAGE; $\left.M_{\mathrm{n}}=6.700 \mathrm{~g} \mathrm{~mol}^{-1}, Ð=1.05\right)$ or cysteamine using thiol-ene chemistry $^{20,29}$ (PEO- $b$-PAGE $\mathrm{NH}_{2}-b$-PtBGE, $53 \%$ functionalization, corresponding to eight units per chain; $M_{\mathrm{n}}=5.750 \mathrm{~g} \mathrm{~mol}^{-1}$, $Ð=1.06)$. In water, these materials undergo self-assembly into coreshell-corona micelles featuring a PtBGE core, a PAGE $\mathrm{NH}_{2}(\mathrm{ENT})$ or PAGE $_{\mathrm{COOH}}$ (ECT) shell, and a PEO corona. In this manner, ECT, ENT or EMT core-shell-corona micelles were prepared by dissolving the respective triblock terpolymer (or mixtures thereof) in THF as non-selective solvent, followed by the addition of water until a $\mathrm{THF} /$ water mixture of 50:50 (v/v) is reached. In case of EMT, we used a mixture of 3.5:1 (ENT:ECT) to ensure a net cationic charge of the 
a

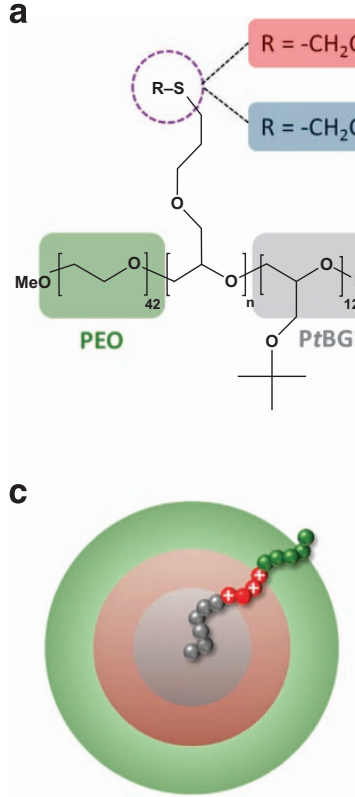

ENT

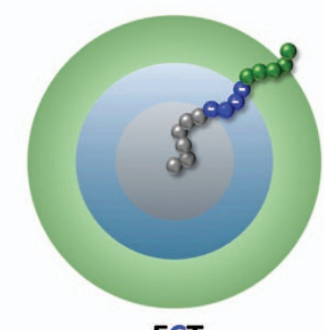

ECT b

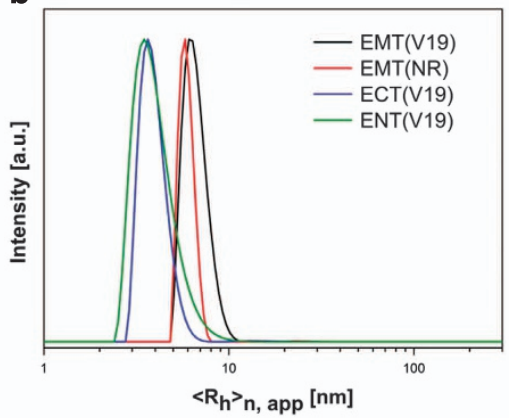

d

e
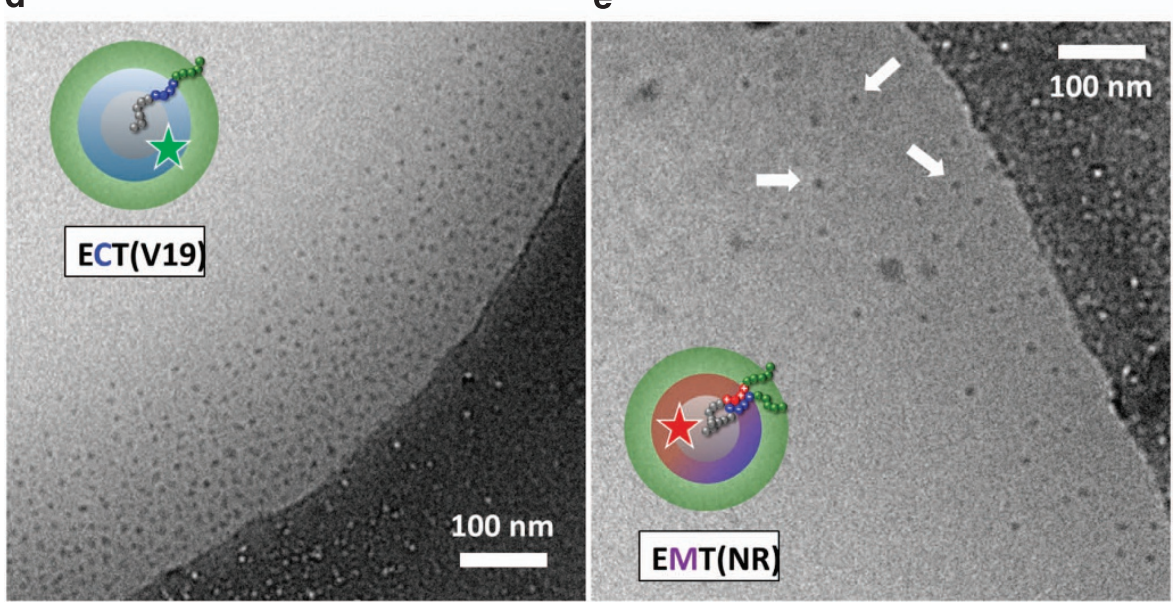

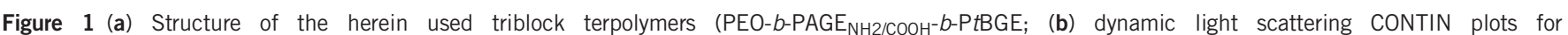
EMT(V19) $\left(<R_{\mathrm{h}}>_{n \text {, app }}=7 \mathrm{~nm}\right.$, solid black line), EMT(NR) $\left(<R_{\mathrm{h}}>_{n}\right.$, app $=8 \mathrm{~nm}$, solid red line), ECT(V19) $\left(<R_{\mathrm{h}}\right\rangle_{n}$, app $=5 \mathrm{~nm}$, solid blue line $)$ and ENT (V19) $\left(<R_{\mathrm{h}}\right\rangle_{n, \text { app }}=5 \mathrm{~nm}$, solid green line; (c) proposed core-shell-corona structure for the micelles used during this study, including the triblock terpolymer chain arrangement; electron micrographs of polyether-based ABC triblock terpolymer micelles, aqueous cryogenic transmission electron micrographs (cryo-TEM) of (d) ECT(V19) and (e) EMT(NR), the arrows highlight representative micelles, the green star resembles V19 and the red star NR, the concentration was $1 \mathrm{gl}^{-1}$. NR, Nile red.

resulting co-micelles. Typical concentrations at this step are $5 \mathrm{gl}^{-1}$ and, subsequently, THF is evaporated and the concentration is adjusted $\left(1-10 \mathrm{~g} \mathrm{l}^{-1}\right)$.

Both NR and V19 (Supplementary Figure S1, see also discussion below) were encapsulated as model substances during this procedure and any excess dye was removed by subsequent filtration. For example, ENT micelles are depicted as ENT(V19) after loading with V19. Both cargos are hydrophobic dyes, although in case of V19 a quaternized aromatic nitrogen imparts permanent cationic charge. Hence, whereas NR is encapsulated predominantly via hydrophobic interactions, also electrostatic interactions have to be taken into account for V19. In one particular case, the $\mathrm{pH}$ was adjusted to $\mathrm{pH}$ 4 during self-assembly to reduce charge density within the $\mathrm{PAGE}_{\mathrm{COOH}}$ block $\left(\mathrm{ECT}_{\mathrm{pH} 4}\right)$. Nevertheless, in all cases the $\mathrm{pH}$ was adjusted to 7 after the evaporation of THF. The proposed micellar structure and characterization data are shown in Figure 1, and hydrodynamic radii (in all cases micelles with $\left\langle R_{\mathrm{h}}\right\rangle_{n}$, app $=5-8 \mathrm{~nm}$ and narrow dispersities were obtained) as well as the corresponding zeta potentials are listed in Table 1. We have shown earlier that these structures feature aggregation numbers $\left(N_{\mathrm{agg}}\right)$ of 150 (ECT) - 320 chains (ENT) approximately using field flow fractionation techniques-although during these measurements the apparent hydrodynamic radii were higher (up to $12 \mathrm{~nm}$ ). ${ }^{21}$ As the later on discussed in vitro and in vivo experiments will lead to-in part-rapid dilution of the micellar solutions, we also investigated the micellar stability by dilution of asprepared stock solutions of ECT using DPH or NR as probe (Supplementary Figure S8). We chose ECT, as this is the most hydrophilic terpolymer investigated here and is thus supposed to show 
Table 1 Size and zeta potential of different micelles

\begin{tabular}{|c|c|c|}
\hline Micelles & $<\mathrm{R}_{h}>_{\mathrm{n}, a p p}(n m)^{a}$ & Zeta potential $(\mathrm{mV})^{\mathrm{b}}$ \\
\hline ECT & 7 & $-41.0 \pm 0.2$ \\
\hline ECT(NR) & 6 & $-38.3 \pm 0.5$ \\
\hline ECT(V19) & 5 & $-17.8 \pm 0.5$ \\
\hline $\mathrm{ECT}_{\mathrm{pH} 4}(\mathrm{~V} 19) @ \mathrm{pH} 4$ & 5 & $-0.5 \pm 0.7$ \\
\hline $\mathrm{ECT}_{\mathrm{pH} 4}(\mathrm{~V} 19) @ \mathrm{pH} 7$ & 5 & $-16.9 \pm 1.0$ \\
\hline ENT & 6 & $23.6 \pm 0.6$ \\
\hline ENT(NR) & 5 & $24.7 \pm 0.9$ \\
\hline ENT(V19) & 5 & $35.1 \pm 0.3$ \\
\hline EMT & 7 & $12.5 \pm 0.5^{21}$ \\
\hline EMT(NR) & 8 & $8.7 \pm 0.1$ \\
\hline EMT(V19) & 7 & $18.7 \pm 0.1$ \\
\hline
\end{tabular}

Abbreviation: NR, Nile red.

aDetermined by dynamic light scattering in deionized water.

Determined using electrophoretic light scattering.

the lowest stability upon dilution, that is, the highest critical micellization concentration (c.m.c.). Dilution was carried out using Krebs-Henseleit buffer as this in our opinion represents conditions, which are at least close to the situation during the in vivo experiments. In case of DPH as probe, the characteristic absorbance at $356 \mathrm{~nm}$ was taken as indication of the location within a hydrophobic environment ${ }^{30}$ and as can be seen in Supplementary Figure S8a and $\mathrm{b}$, the absorption is visible down to $0.005 \mathrm{mg} \mathrm{ml}^{-1}$. Here ECT micelles were prepared using deionized water and diluted using $\mathrm{KH}$ buffer (Supplementary Figure S8a and b), whereas the absorption was hardly visible even at $0.01 \mathrm{mg} \mathrm{ml}^{-1}$ if both preparation and dilution were carried out using $\mathrm{KH}$ buffer. Within the series of concentrations investigated here, a c.m.c. of $0.028 \mathrm{mg} \mathrm{ml}^{-1}$ can be determined (Supplementary Figure S8a). In case of NR as fluorescent probe, a c. m.c. of $0.045 \mathrm{mg} \mathrm{ml}^{-1}$ was determined using the intensity of the emission at $635 \mathrm{~nm}$ (Supplementary Figure S8c).

The micellar shell of EMT is supposed to consist of an interpolyelectrolyte complex between $\mathrm{PAGE}_{\mathrm{NH} 2}$ and $\mathrm{PAGE}_{\mathrm{COOH}}$ as both are highly charged at $\mathrm{pH} 7$. The expected positive net charge in case of EMT is also reflected in zeta potential measurements $(8$ and $18 \mathrm{mV}$, depending on the encapsulated dye). The observed zeta potentials for ECT $(-41 \mathrm{mV})$ and $\mathrm{ECT}(\mathrm{NR})(-38.3 \mathrm{mV})$ are strongly negative, whereas encapsulation of cationic $\mathrm{V} 19$ leads to an increase $(-17.8 \mathrm{mV}$ for $\operatorname{ECT}(\mathrm{V} 19))$. ENT $(23.6 \mathrm{mV})$ and $\operatorname{ENT}(\mathrm{NR})(24.7 \mathrm{mV})$ feature positive zeta potentials, which even increases for ENT(V19) $(35.1 \mathrm{mV})$. Directly after encapsulation at $\mathrm{pH} 4, \mathrm{ECT}_{\mathrm{pH} 4}(\mathrm{~V} 19)$ exhibits almost zero net charge, whereas this decreases to $-16.9 \mathrm{mV}$ upon adjusting the $\mathrm{pH}$ to 7 .

Exemplary, cryogenic transmission electron microscopy for ECT (V19) and EMT(NR) is shown in Figure 1d and e. As can be seen, narrowly dispersed spherical micelles and co-micelles with diameters of $\sim 15 \mathrm{~nm}$ are found, confirming the results from dynamic light scattering.

\section{V19-05048 as surrogate cargo}

In addition to NR, we also used the dye V19. Intravenous injection of $100 \mu \mathrm{g}$ V19 leads to mitochondrial accumulation in mice similar to, for example, Rhodamine 123 or MitoDY-1 (Supplementary Figure S2). ${ }^{24}$ Co-staining in HEK293 cells of V19 with tetramethylrhodamine revealed an increased accumulation of V19 in the mitochondrial matrix. In vivo, a characteristic punctuate staining pattern of mitochondria in kidney tubular epithelial cells and the hepatocytes in the liver is observed. Further the V19 fluorescence signal intensity is stable in liver tissue over an observation period of $90 \mathrm{~min}$, suggesting that V19 is firmly attached to the mitochondria and cannot be metabolized or excreted within the observation period. Therefore, we used V19 as surrogate drug for encapsulation in core-shell-corona micelles as the appearance of a distinct punctuate staining pattern is a direct indication for a release from the micelle.

\section{In vitro studies on micellar uptake mechanism}

We have shown earlier that cellular uptake for ENT and ECT in HEK293 cells was comparable at both 37 and $4{ }^{\circ} \mathrm{C}$, indicating an energy-independent uptake mechanism. ${ }^{21}$ Literature studies suggest that nanomaterials of only few nanometers in size are able to pass cellular membranes by (reversible) pore formation and subsequent translocation. ${ }^{31-33}$ We were therefore interested in further details on uptake mechanisms for ENT, ECT and EMT-with or without model cargo. For this purpose, Raman imaging is a suitable technique to visualize site-dependent distribution of polymeric materials over a cellular background without the need for labels. The triblock terpolymers exhibit distinct Raman spectra as pristine materials (Figure 2a, peak assignment; Supplementary Table S1), which further differ significantly from the cellular background (HepG2 cells) as shown in the difference spectra of cell vesicles and ENT/ECT located nearby (Figure 2b). Complete Raman spectra of the cell vesicles and ENT or ECT micelles are shown in Supplementary Figure S4b (ECT) and S4c (ENT), and are used to identify and localize vesicles (Figure 2c, red) and the micelles (Figure 2c, green). We also applied a spectral unmixing algorithm to the scanned area together with the individual spectra of ECT or ENT to identify the location of the respective micelles without payload. The respective heat map indicates the presence of micelles within vesicles near the cell nucleussuggesting endocytotic uptake and trafficking without endosomal release of ECT (Figure 2d) or ENT (Figure 2e). Using fluorescence microscopy to quantify cellular uptake for micelles loaded with NR (Figure 2f) or V19 (Figure 2g) in vitro, ECT(NR) shows superior uptake in HEK293 cells after $1 \mathrm{~h}$ when compared to ENT and EMT. To test whether protein binding under physiological conditions influences the uptake we performed all experiments in RPMI 1680 media containing $10 \%$ fetal calf serum (R10) and under serumreduced conditions (OptiMEM, OM). In contrast, this trend is reversed for micelles loaded with V19: here ENT(V19) shows the highest uptake within $30 \mathrm{~min}$. Interestingly, the uptake of EMT(V19) increases in the presence of serum, indicating the necessity for certain plasma protein binding (Figure 3g). If the encapsulation of V19 in case of ECT is carried out at $\mathrm{pH} 4\left(\mathrm{ECT}_{\mathrm{pH} 4}(\mathrm{~V} 19)\right)$, the uptake increases both with $10 \%$ serum and under serum-reduced conditions. This suggests differences in micellar surface characteristics depending on the preparation conditions for ECT(V19). If Pitstop-2, a known inhibitor for clathrin-mediated endocytosis, is added cellular uptake decreases for all samples investigated (Figure $2 \mathrm{~h}$ ). However, whereas a decrease of only 10-15\% was observed in the case of ENT(NR) and EMT(NR), the strongest impact was found for ECT(NR). These results indicate a clathrin-independent uptake mechanism for ENT (NR) and EMT(NR) such as direct penetration of the cell wall (translocation), which has been proposed as possible mechanism for cationic nanoparticle penetration and explains previously observed higher cytotoxicity of ENT. ${ }^{21}$

\section{Micellar structure and dye localization}

The nature of the dye seems to have an effect on micellar uptake. Taking into account the structures of NR and V19, the permanent 

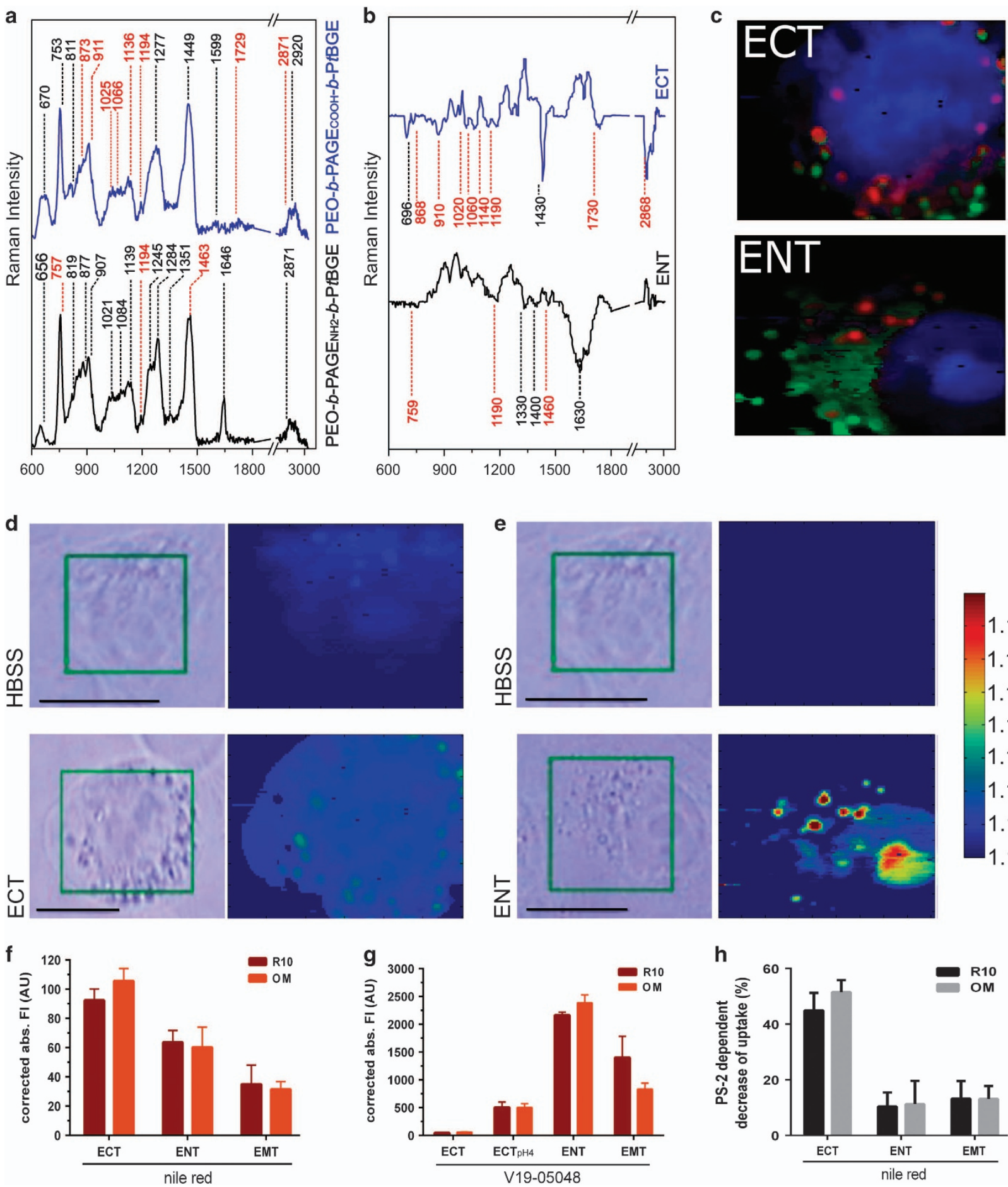

Figure 2 In vitro uptake studies. (a) Raman spectra of micelles and (b) Raman difference spectra of the vesicles and the micelles localizing with the vesicles inside the cell. (c) Raman image of micelles in HepG2 cells after 15 min incubation showing micelles in green and vesicles identified by its specific lipid profile in red. DNA-signal of cells is given in blue. (d, e) HepG2 cells incubated with vehicle (Hanks' balanced salt solution; HBSS), ECT (d) or ENT (e) for 15 min. Raman profile is depicted as false-colored heat map using characteristic micelles' peaks shown in a, scale bar $=20 \mu \mathrm{m}$. Thirty-minute uptake of micelles loaded with Nile red (NR) (f) or V19 (g) in the presence of $10 \%$ fetal calf serum (R10) and under serum-reduced conditions (OM) was quantified using correction factor-adjusted fluorescent intensities from confocal imaging micrographs. (h) Influence of the clathrin inhibitor PS-2 on the uptake of NR-loaded micelles expressed as relative uptake decrease compared to control (w/o PS-2).

positive charge of V19 leads to strong electrostatic interactions during encapsulation within ECT (or EMT), at least more pronounced in comparison to NR. Hence, an exclusive localization of V19 in the PtBGE core seems unlikely and a colocalization within core and shell can be anticipated, depending on the $\mathrm{pH}$ and charge density in the $\mathrm{PAGE}_{\mathrm{COOH}}$ block. As promising and noninvasive technique to investigate this, we identified Raman spectroscopy - this methodology probes bond vibrations, and is further sensitive to conformational 
a
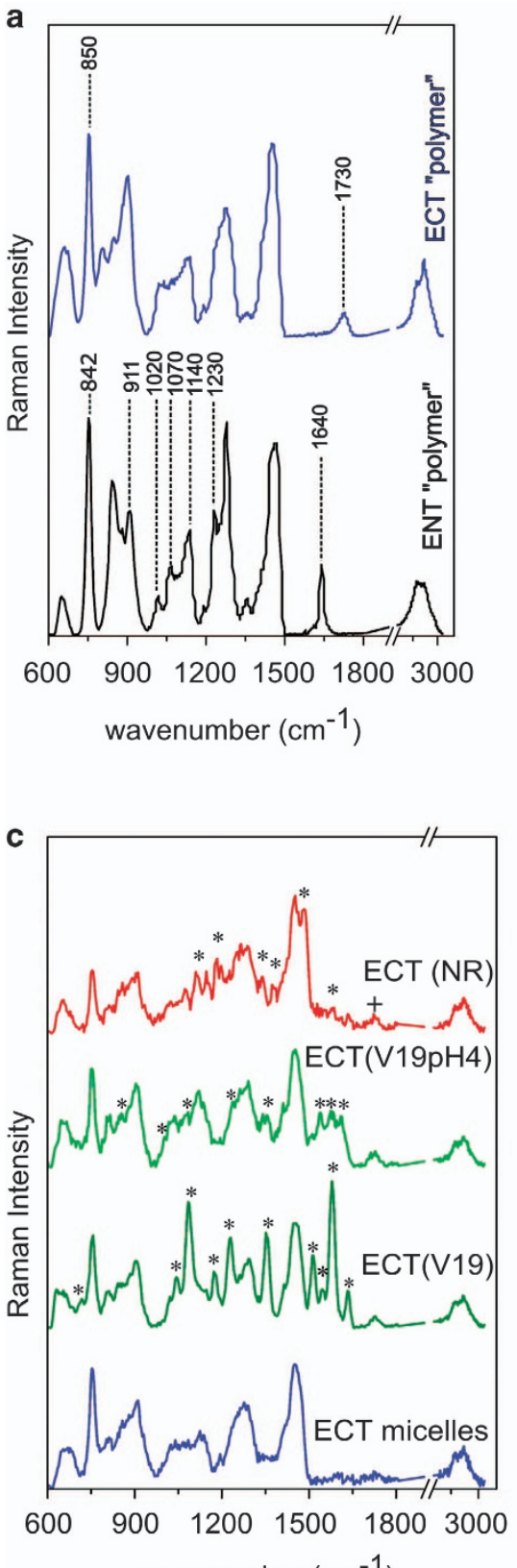

b
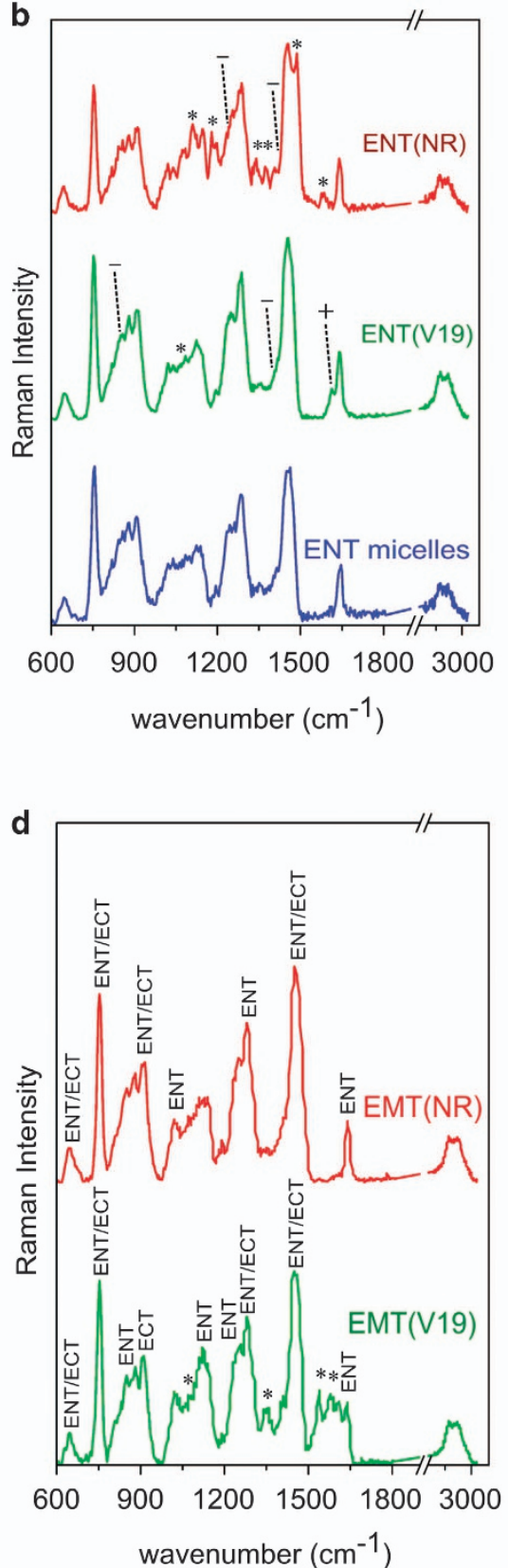

e
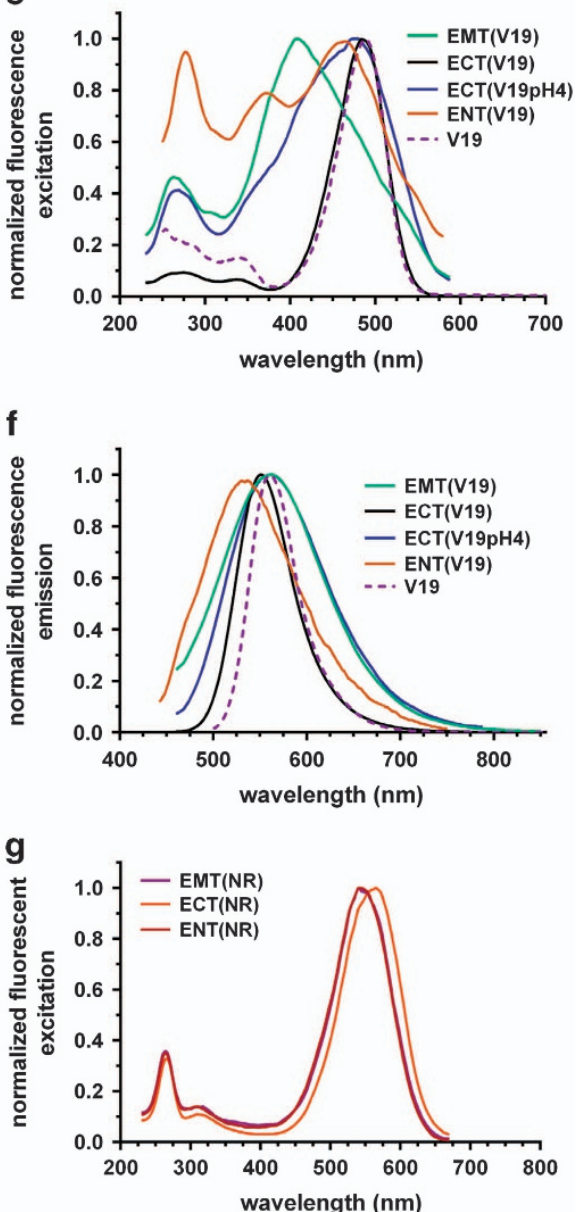

h

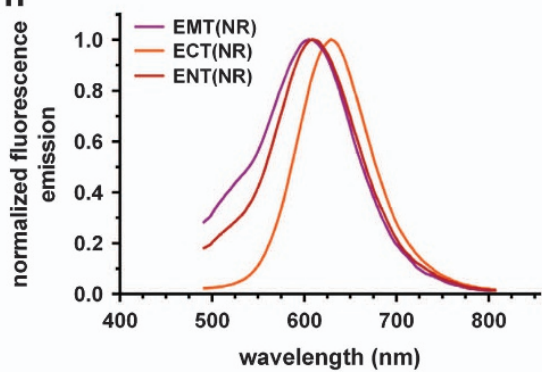

Figure 3 Raman and fluorescence spectra of the herein-used ABC triblock terpolymers and micelles. Raman spectra of the neat materials (a) and after selfassembly into core-shell-corona micelles ENT (b), ECT (c) or EMT (d) - after loading with V19, NR or without cargo. Raman signals for the respective dye are marked with an asterisk $\left({ }^{*}\right),{ }^{\prime}$ ' refers to additional and '-' to suppressed vibrations. In case of EMT the Raman peaks belonging to the polymers ECT and ENT are further highlighted. (e-h) Fluorescence excitation and emission of different micelles after encapsulation of V19 or NR.

changes of the molecule due to changes in its surroundings, enabling the detection of changes in the localization of the payload (Figure 3). Raman spectra of the bulk triblock terpolymers (Supplementary Figure S4a) show the characteristic spectrum for each building block. Differences are already visible when comparing pristine ENT and ECT and micelles (Figure $3 \mathrm{a}-\mathrm{c}$ ). The most prominent differences are observed in the Raman peaks of $-\mathrm{NH}_{2}\left(1640 \mathrm{~cm}^{-1}\right.$, ENT) and $-\mathrm{COOH}\left(1730 \mathrm{~cm}^{-1}, \mathrm{ECT}\right)$. The $\mathrm{CH}_{2}$ rocking of $\mathrm{PEO}$ was not detectable for pristine $\mathrm{PEO}-b-\mathrm{PAGE}_{\mathrm{NH} 2}-b-\mathrm{P} t \mathrm{BGE}$ but was for the respective micelle. Same holds true for the $\mathrm{C}-\mathrm{O}$ stretching vibration for $\mathrm{PEO}$ in case of ECT, and the $-\mathrm{COOH}$ vibration is completely suppressed indicating conformational changes in the PEO group of these micelles (Figure 3c; Supplementary Table S1).

The Raman signatures of $\mathrm{V} 19$ and NR are depicted in Supplementary Figure S4d and differ upon encapsulation within ENT, ECT or EMT-suggesting changes in the environment for the respective dye (Figure $3 \mathrm{~b}-\mathrm{d}$ ). Notably, the very prominent NR Raman bands at 1484 and $1586 \mathrm{~cm}^{-1}$ are prominently visible in ENT(NR) and ECT(NR), and are marked with ' ' along with other visible NR vibrations (Figure $3 \mathrm{~b}$ and c) - but are completely suppressed for EMT (Figure 3d). Prominent Raman bands for V19, also marked with '*', are suppressed in case of ENT(V19), weakly visible for $\mathrm{ECT}_{\mathrm{pH} 4}(\mathrm{~V} 19)$, 


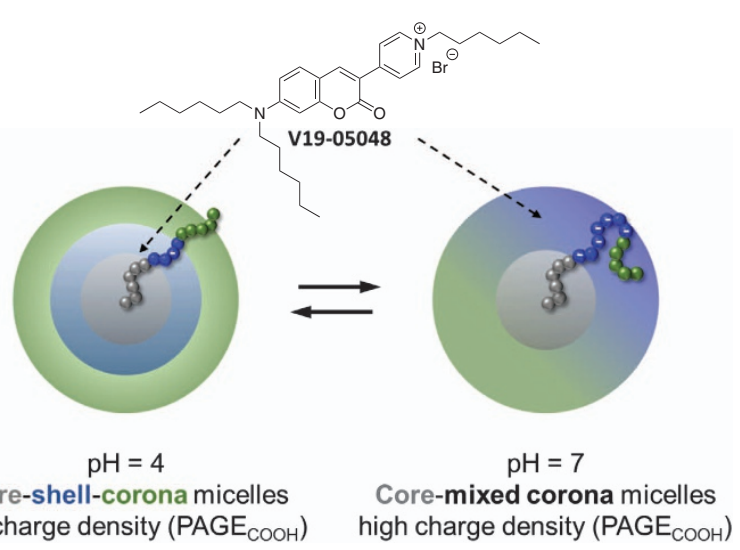

low charge density $\left(\mathrm{PAGE}_{\mathrm{COOH}}\right)$

Figure 4 Proposed micellar structure for ECT (PEO- $b$-PAGE $\mathrm{COOH}^{-} b$-PtBGE) during encapsulation studies at $\mathrm{pH} 4$ (left) and 7 (right); structure and implications for the location of the dye V19 upon encapsulation are indicated with dotted black arrows.

but prominently observed in ECT(V19). Interestingly, the encapsulation of V19 or NR also affects vibrational bands of the micelles, and these alterations are marked with ' + ' if an additional band is observed or ' - ' if a band is suppressed (Figure $3 \mathrm{~b}$ and c). Besides suppression of two Raman signals at 1234 and $1414 \mathrm{~cm}^{-1}$ in case of ENT(NR) and 842 and $1418 \mathrm{~cm}^{-1}$ in case of ENT(V19), an additional band at $1613 \mathrm{~cm}^{-1}$ can be observed for ENT(V19). For ECT(NR), ECT(V19) and $\mathrm{ECT}_{\mathrm{pH} 4}(\mathrm{~V} 19)$, the $\mathrm{COO}^{-}$vibration of ECT at $1730 \mathrm{~cm}^{-1}$, which is suppressed in ECT micelles is visible (Figure 3c). Also, encapsulation of V19 at pH4 leads to a distinct vibrational profile compared to ECT (V19) (Supplementary Figure S4e, this is also supported by Raman spectra of both $\mathrm{ECT}(\mathrm{V} 19)$ and $\mathrm{ECT}_{\mathrm{pH} 4}(\mathrm{~V} 19)$ in Krebs-Henseleit buffer solutions-Supplementary Figure S7). In case of EMT(V19), characteristic Raman signals for V19 are observed at 1090, 1360, 1540 and $1580 \mathrm{~cm}^{-1}$, also additional peaks for ECT are present (Figure 3d). In summary, Raman spectra depict changes for the terpolymers and the corresponding micelles depending on the encapsulated cargo. Especially, vibrational changes associated with PEO and PAGE indicate alterations in their conformation.

Further evidence for our hypothesis concerning different localization of V19 within the micelles is provided by changes in its excitation and emission spectra. For EMT(V19), an excitation maximum at $397 \mathrm{~nm}$ was found, which is shifted to $489 \mathrm{~nm}$ for ECT(V19) and $492 \mathrm{~nm}$ for $\mathrm{ECT}_{\mathrm{pH} 4}(\mathrm{~V} 19)$ - both being close to the excitation maximum of the pristine dye at $489 \mathrm{~nm}$. In the case of ECT(V19) the excitation spectrum is significantly broadened, and the excitation is increased toward shorter wavelengths compared to $\mathrm{ECT}_{\mathrm{pH} 4}(\mathrm{~V} 19)$. This suggests that the environment of the dye has been changed, presumably due to the encapsulation process. Also, a second and weaker excitation band at $385 \mathrm{~nm}$ can be found for EMT(V19) and $\mathrm{ECT}(\mathrm{V} 19)$, which is nearly completely suppressed in $\mathrm{ECT}_{\mathrm{pH} 4}(\mathrm{~V} 19)$. These changes are also partly reflected in the corresponding emission spectra, where EMT(V19) and ECT(V19) feature broader emission spectra compared to $\mathrm{ECT}_{\mathrm{pH} 4}(\mathrm{~V} 19)$, the latter being almost unchanged if compared to the dye itself (Figure 3f). On the contrary, ENT loaded with NR exhibited a bathochromic shift in its excitation and emission compared to EMT(NR) and ENT(NR) (Figure $3 g$ and $h$ ).

From these data, a similar environment-dependent localization of V19 for EMT(V19) and ECT(V19) can be deduced, whereas this significantly changes upon encapsulation at $\mathrm{pH}$ 4-at conditions where the charge density within the $\mathrm{PAGE}_{\mathrm{COOH}}$ block is reduced. On the basis of the structure and sequence of the used PEO- $b$ -
$\mathrm{PAGE}_{\mathrm{COOH}}-b$-PtBGE, two different chain conformations and, hence, micellar morphologies can be anticipated (Figure 4). At pH 4, charge density in $\mathrm{PAGE}_{\mathrm{COOH}}$ is low and the micelle can be best described as core-shell-corona structure-electrostatic interaction between V19 and $\mathrm{PAGE}_{\mathrm{COOH}}$ is reduced and we expect V19 to colocalize mostly at the interface between $\mathrm{P} t \mathrm{BGE}$ core and the $\mathrm{PAGE}_{\mathrm{COOH}}$ shell as some negative charges are still present. The situation appears to be different at $\mathrm{pH} \mathrm{7}$; here, almost complete ionization can be expected for $\mathrm{PAGE}_{\mathrm{COOH}}$ and electrostatic repulsion induces chain stretching. This is facilitated as the PEO segment has a relatively low degree of polymerization and might lead to a situation where $\mathrm{PAGE}_{\mathrm{COOH}}$ is partly exposed at the micellar surface and micelles with a mixed corona consisting of $\mathrm{PEO}$ and $\mathrm{PAGE}_{\mathrm{COOH}}$ are formed instead of coreshell-corona structures. Electrostatic complexation of V19 by PAGE$\mathrm{COOH}$ is more pronounced and this can lead to localization of V19 in the mixed corona. This is also reflected in the Raman spectrum of ECT(V19), where characteristic Raman bands of V19 (Figure 3c, marked with '‘') are more pronounced if compared to $\mathrm{ECT}_{\mathrm{pH} 4}(\mathrm{~V} 19)$ and the spectrum rather resembles the bulk V19 data (Supplementary Figure S4d). Comparable chain rearrangements have been shown for BMAAD triblock terpolymer micelles of 120-200 nm size at different pH values. ${ }^{34}$ However, such subtle morphological changes are difficult if not impossible to verify using electron microscopy for such small nanostructures (sub $30 \mathrm{~nm}$ ). Furthermore, the two situations depicted in Figure 4 represent the extreme morphological states, and the true situation during encapsulation might be located in between. Nevertheless, at this point we propose that upon encapsulation at $\mathrm{pH} 4$ the model dye V19 is mostly localized at the interface between PtBGE and $\mathrm{PAGE}_{\mathrm{COOH}}$ shell, whereas comparable experiments at $\mathrm{pH} 7$ lead to micelles where V19 is located predominantly within the PAGE $\mathrm{COOH}_{\mathrm{H}}$ block present in both shell and (mixed) corona. Although the conformation of the $\mathrm{PAGE}_{\mathrm{COOH}}$ block will inevitably change upon re-adjustment of the $\mathrm{pH}$ to 7.4 after encapsulation even for ECT $\left(\mathrm{V} 19_{\mathrm{pH} 4}\right)$, this will drastically affect the localization and accessibility of V19 within the resulting micellar nanostructures.

One of the most surface-sensitive systems are cells, especially in an organism where the surface properties of any foreign matter may affect interaction with cells, induction of immune responses, or metabolism and clearance. Many different cell types are present in the liver, an organ with primary metabolic, foreign particle and xenobiotic clearance, and immunological functions. If the conformational changes truly alter the surface of the triblock terpolymer micelles as suggested by the results above, we postulate differences will occur both in the localization and elimination of the micelles after administration (Figure 4).

\section{Kinetics and uptake of micelles in vivo}

We used intravital microscopy to analyze the distribution of micelles within a tissue or organ. Besides localization, information on uptake kinetics can be simultaneously extracted from a time series. In the case of hepatocytes, the dominant cell type in the liver, handling of micelles after uptake can be visualized. Once a xenobiotic enters hepatocytes it is usually subjected to biotransformation and secretion into the bile. This can not only be visualized but can also be subjected to kinetic analysis to shed light on pharmacokinetics. To understand the effects of shell modifications and the impact of the payloads on pharmacokinetics we used male Wistar rats, a standard model to analyze pharmacokinetics. Surgical techniques and anatomical features of the rat, such as the lack of a gallbladder, which allows collecting of biliary outflow directly, make it a suitable model to analyze plasma disappearance rate and secretion via the liver and the kidney. Rats used in the study had a body weight of maximal $24 \mathrm{~g}$, resulting in a 
a

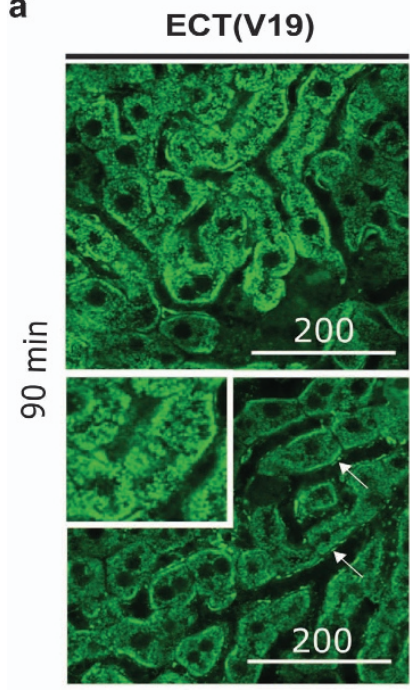

ECT(V19pH4)
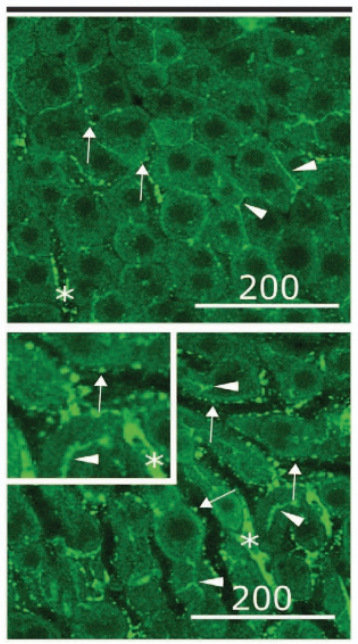

b
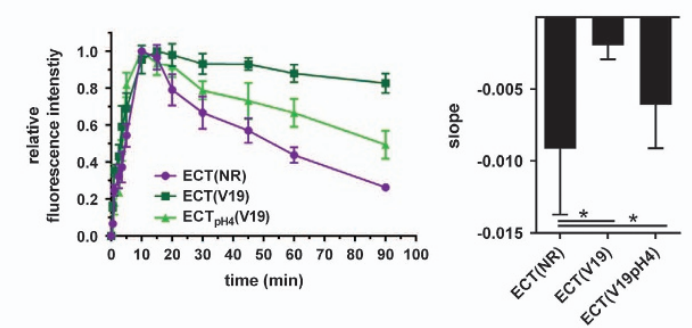

ECT(NR)

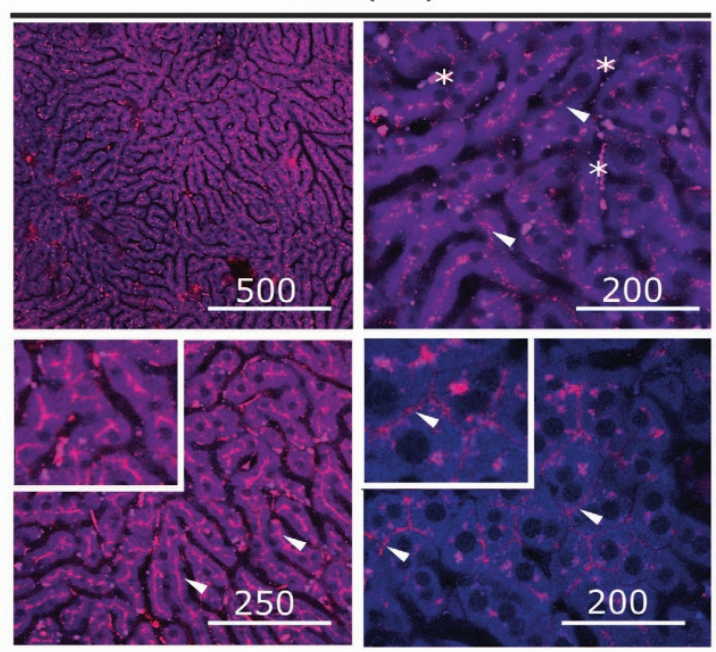

C
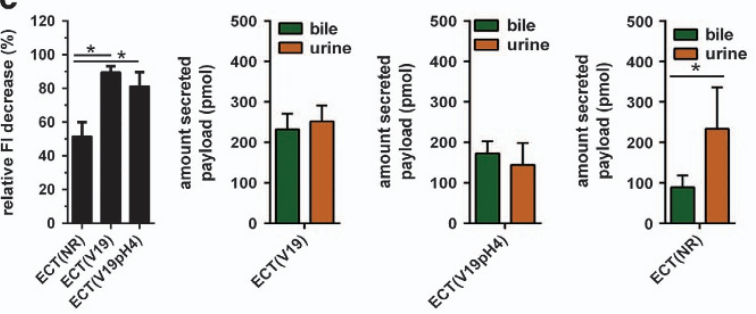

d
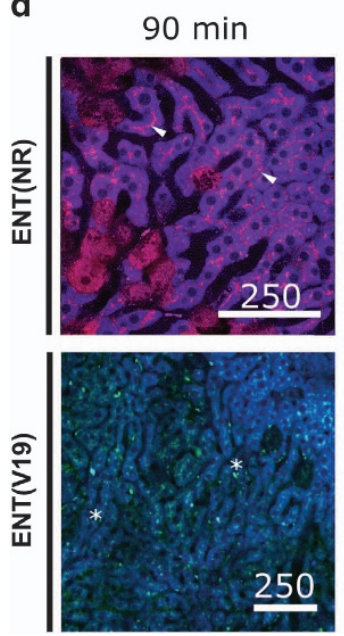

$90 \mathrm{~min}$
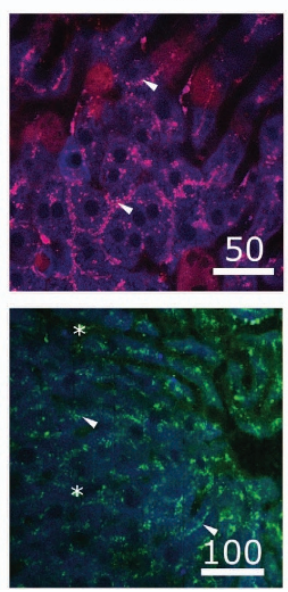

$90 \mathrm{~min}$
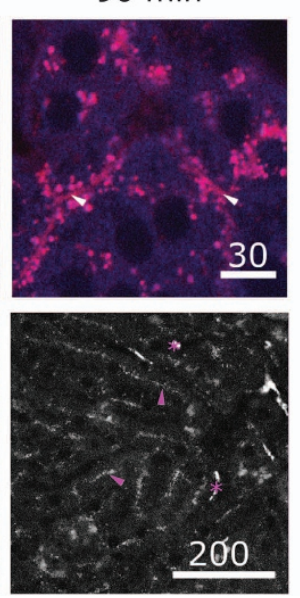

e
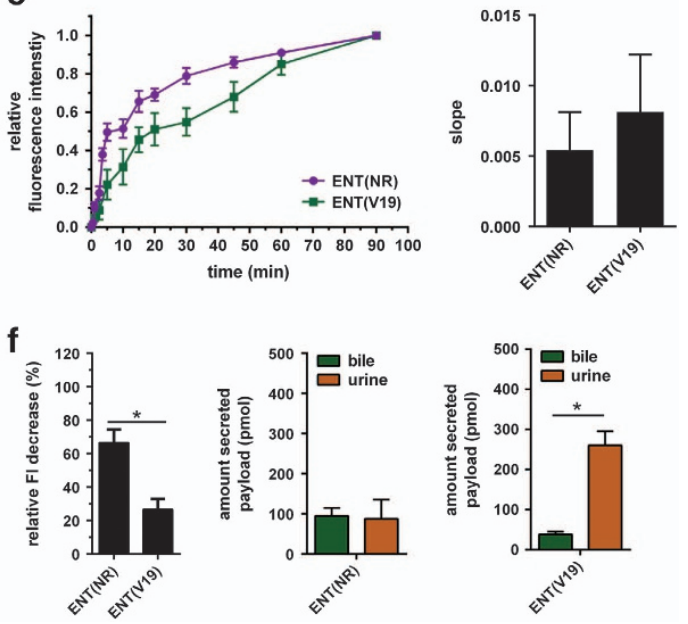

Figure 5 In vivo analysis of pharmacokinetic/pharmacodynamic of various ECT- and ENT-based micelles. (a, d) Intravital microscopy of ECT(V19), ECT ${ }_{\mathrm{pH} 4}$ (V19) or ENT(V19) (green), and ECT(NR) or ENT(NR) (red) distribution pattern in the murine liver after 90 min; scale bar: $200 \mu m$ (a, b: first image upper panel and complete lower panel), $50 \mu \mathrm{m}$ (b: second and third images upper panel). Autofluorescence of NAD(P)H is exploited to visualize liver tissue in some images (blue). Triangles (white or pink) mark a few canaliculi structures; arrows highlight a few areas of uptake in endothelia cells (dotted accumulations along the vasculature); stars point to a few Kupffer cells, which have taken up micelles (bright, big spots). (b, d) Results of plasma disappearance rate measurements in rats: relative FI-plasma decrease correlates inversely with circulation time, further hepatic and renal excreted payload in bile or urine, respectively, is quantified via standard curves. (c, f) Relative fluorescent intensity (FI) change in hepatocytes analyzed by intravital microscopy. Data points in b, $\mathbf{c}$, e and $\mathbf{f}$ depicted as mean \pm s.e.m. ${ }^{*} P<0.05$, unpaired t-test between groups.

blood volume of $1.92 \mathrm{ml}$ (usually, a maximum of $8 \%$ body weight consists of blood). As we injected $200 \mu \mathrm{g}$ sample in $200 \mu \mathrm{l}$ sterile, 0.9 $\%$ sodium chloride solution, a local concentration of at least $0.094 \mathrm{mg} \mathrm{ml}^{-1}$ for the different triblock terpolymers can be assumed $(200 \mu \mathrm{g}$ within $2.12 \mathrm{ml})$. This renders the micellar concentration within this set of experiments close to but still above the values determined for the c.m.c. of the most hydrophilic triblock terpolymer (ECT, see discussion above).

We first investigated micelles from $\mathrm{PEO}-b$-PAGE $\mathrm{COOH}^{-} b$-PtBGE (ECT). Considering the rather poor uptake of ECT(V19) in vitro it is surprising that in vivo as early as $1 \mathrm{~min}$ after injection a strong interaction of ECT(V19) with the vascular lining in liver tissue can be 

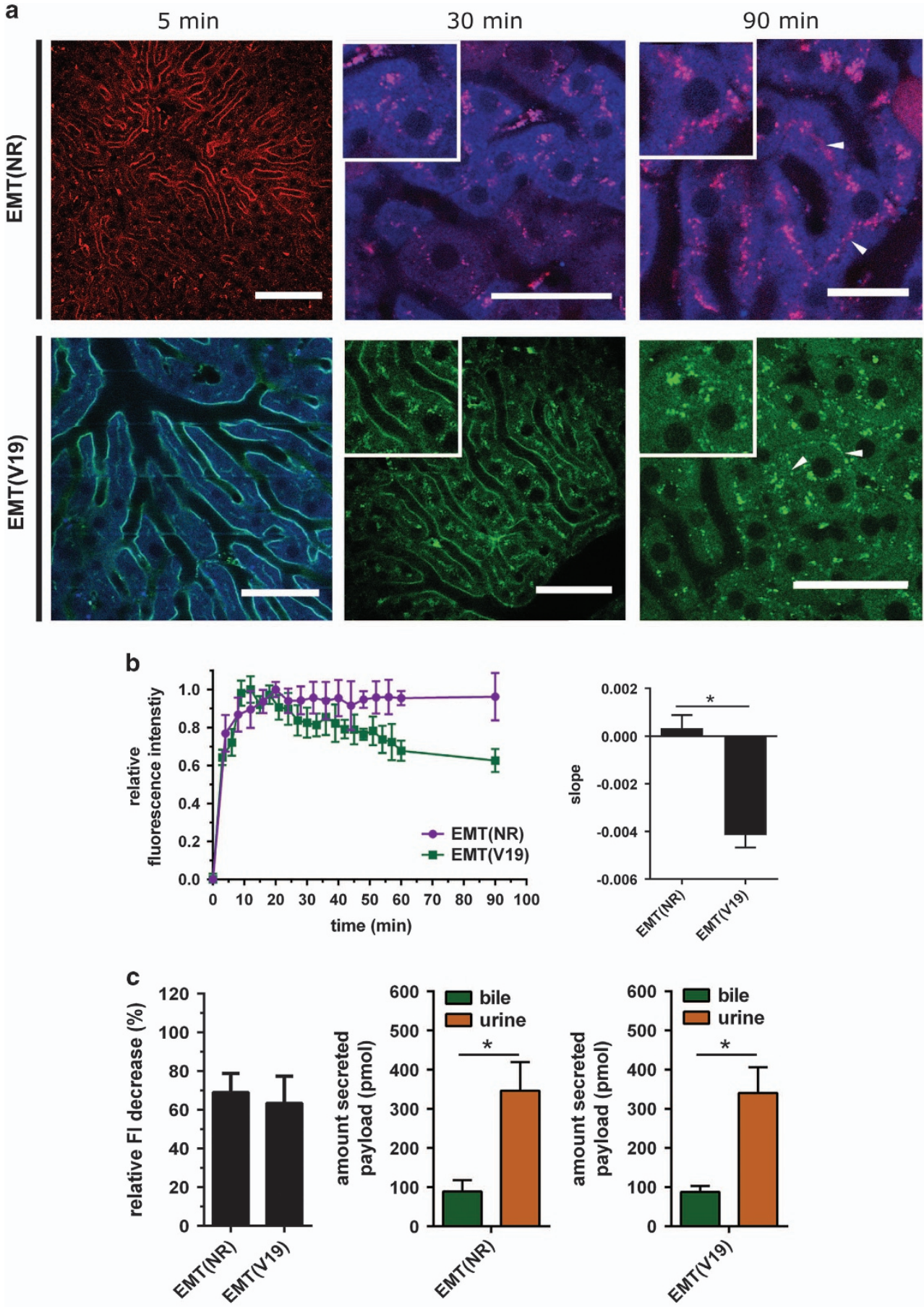

Figure 6 In vivo analysis of EMT. (a) Intravital microscopy of EMT(V19) (green) and EMT(NR) (red) distribution pattern in the murine liver after 5, 30 and 90 min. Scale bar: $200 \mu \mathrm{m}$. Autofluorescence of $\mathrm{NAD}(\mathrm{P}) \mathrm{H}$ is exploited to visualize liver tissue in some images (blue). (b) Relative fluorescent intensity (FI) change in hepatocytes analyzed by intravital microscopy. (c) Results of plasma disappearance rate measurements in rats: relative $\mathrm{Fl}$ plasma decrease correlates inversely with circulation time, further hepatic and renal excreted payload in bile or urine, respectively, is quantified via standard curves. Triangle (white) mark a few canaliculi structures. Data points in $\mathbf{b}, \mathbf{c}$ depicted as mean \pm s.e.m. ${ }^{*} P<0.05$, unpaired $t$-test between groups. NR, Nile red.

observed (Figure 5a). This effect spread over the acinus and led to a punctuate staining pattern in hepatocytes after $10 \mathrm{~min}$, similar to free dye V19 as observed earlier (Supplementary Figure S3) indicating that V19 is able to dissociate from ECT and pass through membranes to interact with mitochondria in vivo driven by the negative membrane potential of this organelle, ${ }^{24,35}$ which could not be observed for the other micelles tested. This pattern was also observed after $90 \mathrm{~min}$ suggesting long-term retention in polarized mitochondria. In contrast, $\mathrm{ECT}_{\mathrm{pH} 4}(\mathrm{~V} 19)$ does not lead to such punctuate staining pattern after 15 or even after $90 \mathrm{~min}$ (that is, is not released as assumed before), supporting our earlier conclusions regarding a difference in location of V19 within the nanocarriers. In this case, the cytosol of hepatocytes appears evenly stained and canalicular structures are visible suggesting an excretion of $\mathrm{ECT}_{\mathrm{pH} 4}(\mathrm{~V} 19)$ via the hepato-biliary route. Further, 
$\mathrm{ECT}_{\mathrm{pH} 4}(\mathrm{~V} 19)$ is effectively cleared by Kupffer cells and liver sinusoidal endothelia.

When replacing V19 with NR, Kupffer cells appear to be slightly stained by ECT(NR), and perinuclear accumulation of vesicles is visible. Similar to $\mathrm{ECT}_{\mathrm{pH} 4}(\mathrm{~V} 19)$, canaliculi are stained with $\mathrm{NR}$, however it remains unclear whether NR has been released and is subsequently secreted or whether this accounts for the fate of entire micelles (Figure 5a). All ECT-based micelles show a decrease in fluorescence intensity in liver tissue over time with different decay slopes (Figure 5b), which is in accordance with fluorescence intensity decrease observed in plasma over $90 \mathrm{~min}$ and concomitant hepato-biliary secretion. Thus, it is apparent that ECT(NR) circulates significantly longer than ECT(V19). Despite their distinct characteristics $\mathrm{ECT}(\mathrm{V} 19)$ and $\mathrm{ECT}_{\mathrm{pH} 4}(\mathrm{~V} 19)$ have a rather similar circulation time but differ in their excretion route-here ECT(V19) is predominantly excreted via the kidneys, whereas $\mathrm{ECT}_{\mathrm{pH} 4}(\mathrm{~V} 19)$ appears to be more rapidly excreted in bile (Figure $5 \mathrm{c}$; Supplementary Figure S6).

Usually, micelles and other particles featuring a PEO corona have a long circulation time due to reduced cellular interactions. In the case of ECT, ECT(NR) is rapidly cleared by the kidney and shows relatively little clearance by hepatocytes in the liver. In addition, no interaction with immune cells was observed for any nanostructures with an outer PEO layer. This was not the case for ECT(V19) and $\mathrm{ECT}_{\mathrm{pH} 4}(\mathrm{~V} 19)$. Here presumably a heterogeneous micellar surface is presented in the case of ECT(V19), together with a colocalization of V19 within shell and corona, leading to release and rapid accumulation in liver tissue. However, encapsulation of V19 at $\mathrm{pH} 4$ seems to influence surface characteristics and dye localization, as in vivo data and biotransformation change upon investigation of $\mathrm{ECT}_{\mathrm{pH} 4}(\mathrm{~V} 19)$. At the current stage, we expect the micelles to be stable under the conditions reported here based on assessments of the c.m.c. in Krebs-Henseleit buffer to mimic physiological conditions as well as dynamic light scattering and cryogenic transmission electron microscopy experiments (Supplementary Figure S7).

ENT(NR) and ENT(V19) both show perinuclear and canalicular staining but no observable uptake in liver sinusoidal endothelia cells and Kupffer cells. ENT(NR) further shows prominent vesicular accumulation around the canaliculi (Figure 5d). In all cases, micelles based on ENT whatever the payload feature steadily increasing concentrations in hepatic tissue over $90 \mathrm{~min}$ as demonstrated by fluorescence intensity measurements (Figure 5e), and again plasmadisappearance rates in rats support these finding by long plasma circulation times and, especially for $\mathrm{ENT}(\mathrm{V} 19)$, only little biliary secretion. While the payload V19, compared to NR, decreases circulation time for ECT micelles it seems to significantly prolong the latter in case of ENT. Even more, ENT(V19) shows the highest retention in plasma over 90 min of all samples investigated (Figure 5f; Supplementary Figure S6).

EMT(NR) and EMT(V19) are similar in their hepatic staining pattern compared to ECT and ENT. Both show uptake by Kupffer cells and very weak staining of canaliculi (Figure 6a). Finally, both EMT(NR) and EMT(V19) feature comparable hepato-cellular linings after $5 \mathrm{~min}$, indicating similar membrane interaction. While this lining vanishes within $30 \mathrm{~min}$ for EMT(NR), and perinuclear as well as canalicular vesicles become visible, it lasted longer in case of EMT(V19), but also disappears after $90 \mathrm{~min}$ (Figure 6a). Regarding uptake kinetics, the fluorescence intensity of EMT(NR) reaches a plateau in liver tissue after $20 \mathrm{~min}$, whereas that of EMT(V19) already decreases after $10 \mathrm{~min}$ (Figure 6b). However, plasma-disappearance rates of both EMT micelles appear rather similar (Figure 6c; Supplementary Figure S6), accompanied by rather short circulation times, especially if compared to ENT(V19) and $\mathrm{ECT}(\mathrm{NR})$.

\section{DISCUSSION}

Factors influencing the biodistribution of polymeric nanoparticles are hardly understood, especially when it comes to effects of size, surface chemistry or charge density. The herein presented ABC triblock terpolymer platform provides straightforward access to nanocarriers with precisely tunable chemistry, surface charge, as well as size and shape (perspectively in the latter case). Our combined Raman, spectroscopic and pharmacokinetic data indicate that the location of both model drugs, NR and V19, differs within nanostructures, more pronounced in case of V19. We hypothesize that V19 exhibits stronger electrostatic interactions with ECT and is thus at least partially localized in the micellar shell of ECT(V19). Encapsulation at lower $\mathrm{pH}$ seems to balance this effect as demonstrated for $\mathrm{ECT}_{\mathrm{pH} 4}(\mathrm{~V} 19)$. In that regard, label-free Raman imaging provided information on the actual terpolymer conformation and additional insight into the intracellular fate of the micelles.

Previous in vitro studies already demonstrated that alterations in shell composition (especially charge and charge density) of polyether-based $\mathrm{ABC}$ triblock terpolymer micelles had great impact on uptake efficacy and cellular interactions. ${ }^{21}$ The herein-presented data now support these claims with in vivo data on biodistribution and renal versus biliary excretion. By using NR and V19 as model payloads, micellar pharmacokinetics and hepatic clearance were shown to depend not only on the effective shell charge but also on the conditions applied during encapsulation as both the middle segments of ECT and ENT feature characteristics of weak polyelectrolytes. Thus, to understand the pharmacokinetic/pharmacodynamic it was required to not only analyze the circulation time but also observe the interaction with professional phagocytes as well as alternative routes of plasma elimination, a process in which the liver with its differing cell populations has a prominent role. Specifically, whether or not drug carriers are taken up either by parenchymal, or non-parenchymal liver cells (sinusoidal endothelial cells or Kupffer cells) or both has a practical importance as this would indicate either a primary biliary route of excretion or, conversely, immune-modulatory effects of the respective nanocarriers or their payload when they are taken up primarily by Kupffer cells. In that respect, many effects might trigger phagocytosis by Kupffer cells in the liver, ${ }^{36}$ and probably the best-characterized effect is their complement activation facilitating opsonization, which depends on charge, size and shape as well as surface chemistry. 37,38

We have shown that free (or released) V19 accumulates in mitochondria and thereby serves as an inherent indicator for its intracellular availability. Interestingly, the release of V19 and therefore its availability was depending on the localization within the herein-used core-shell-corona micelles, which again depended on the $\mathrm{pH}$ of the solutions during encapsulation, pointing out the relevance of charge-dependent carrier-cargo interaction during this process. Although the different localization of V19 in the core/shell $(\mathrm{ECT}(\mathrm{V} 19))$ and shell/corona $\left(\mathrm{ECT}\left(\mathrm{V} 19_{\mathrm{pH}}\right)\right)$ seems like a small alteration, we observed significant differences concerning (Raman) spectroscopic signature, micellar characteristics such as zeta potential and pharmacokinetic profile in subsequent in vivo experiments. Even further, the encapsulation conditions also affect the homogeneity and functionality of the PEO corona, as phagocytosis of micelles by Kupffer cells in the liver occurred de novo for 
ECT $\left(\mathrm{V} 19_{\mathrm{pH} 4}\right)$, which was not observable before for ECT(V19). Such at first glance subtle differences might result in significant off-target actions and might determine whether such micelles can be applied safely as a carrier to deliver drugs selectively to hepatic parenchymal cells. $^{25}$

On the basis of spectroscopic data and in vitro/in vivo experiments, we propose two different chain conformations of $\mathrm{PEO}-b-\mathrm{PAGE} \mathrm{COOH}^{-}$ $b$-PtBGE during encapsulation, depending on the solution $\mathrm{pH}$, and with that also the localization of V19 within the micelles differs. These effects in turn affect cell-type-specific release within the liver acinus. These physicochemical properties are reflected in changes in circulation time, clearance by the reticuloendothelial system, as well as altered cellular uptake. Although Raman spectroscopy strongly suggests a different localization of V19 within the micelles upon different encapsulation conditions, with the current set of tools a direct structural proof of this hypothesis is unfortunately not possible. Nevertheless, this will serve as starting point for systematic alterations of the polyether toolbox in terms of functionalization and molar mass. In combination with the herein-used set of techniques for an indirect analysis of surface homogeneity, this will provide further information and allows a direct evaluation of the clinical applicability of such systems in the future.

Importantly, the present study demonstrates differences in pharmacokinetics and biodistribution not only dependent on (charge of) the nanocarrier but also dependent on the properties of the cargo drugs suggesting that nanoformulated carrier systems must be selected or adapted to the specific drugs that should be delivered to achieve optimal delivery to a given tissue.

\section{CONFLICT OF INTEREST}

The authors declare no conflict of interest.

\section{ACKNOWLEDGEMENTS}

We acknowledge BMBF funding via the Integrated Research and Treatment Center (CSCC, FKZ 01EO1502) and the DFG-funded Collaborative Research Centre PolyTarget (SFB 1278, Project C03). We thank Markus J Barthel for synthesizing some of the triblock terpolymers, Mark Billing for the preparation of some of the micellar solutions and Michael Schmitt (FSU Jena) for fruitful discussions. FHS gratefully acknowledges funding from the DFG

(SCHA1640/7-1), the Thuringian Ministry for Education, Science, and Culture (TMBWK, \#B515-10065), and the Fonds der Chemischen Industrie (FCI). MB acknowledges the Federal Ministry of Education and Research (Project: smartdye-livery, FKZ 13N13416) for funding. AR acknowledges EU funding via 'HemoSpec' (FP 7, CN 611682). The transmission electron microscopy facilities were funded by DFG (German Research Foundation) and EFRE (European Fund for Regional Development). We are further grateful to Dyomics GmbH for continuous support.

Author contributions: ATP and FHS conceived the experiments. ATP conducted and analyzed in vitro and in vivo studies and prepared figures. AR planned and conducted Raman imaging and analysis, prepared figures, and was involved in writing the manuscript. ACR planned in vitro experiments, conducted cell culture experiments, and was involved in writing the manuscript. MvdL performed electron microscopy of ECT and EMT and MvdL and CR determined the $\mathrm{cmc}$ of ECT. MBu performed plasma disappearance rate measurements. JH Performed spectral and data analysis. CP and CR prepared micelles, performed DLS measurements, and were involved in writing the manuscript. UN planned Raman studies and was involved in writing the manuscript. FHS and MB supervised experiments and ATP, FHS, and MB wrote the manuscript.

\section{PUBLISHER'S NOTE}

Springer Nature remains neutral with regard to jurisdictional claims in published maps and institutional affiliations.
1 Recknagel, P., Gonnert, F. A., Westermann, M., Lambeck, S., Lupp, A., Rudiger, A., Dyson, A., Carré, J. E., Kortgen, A., Krafft, C., Popp, J., Sponholz, C., Fuhrmann, V., Hilger, I., Claus, R. A., Riedemann, N. C., Wetzker, R., Singer, M., Trauner, M. \& Bauer, M. Liver dysfunction and phosphatidylinositol-3-kinase signalling in early sepsis: experimental studies in rodent models of peritonitis. PLoS Med. 9, e1001338 (2012).

2 Tibbitt, M. W., Dahlman, J. E. \& Langer, R. Emerging frontiers in drug delivery. J. Am. Chem. Soc. 138, 704-717 (2016)

3 Zhang, S., Gao, H. \& Bao, G. Physical principles of nanoparticle cellular endocytosis. ACS Nano 9, 8655-8671 (2015).

4 Zhu, M., Nie, G., Meng, H., Xia, T., Nel, A. \& Zhao, Y. Physicochemical properties determine nanomaterial cellular uptake, transport, and fate. Acc. Chem. Res. 46, 622-631 (2013).

5 Rivera-Gil, P., Jimenez de Aberasturi, D., Wulf, V., Pelaz, B., del Pino, P., Zhao, Y., de la Fuente, J. M., Ruiz de Larramendi, I., Rojo, T., Liang, X. J. \& Parak, W. J. The challenge to relate the physicochemical properties of colloidal nanoparticles to their cytotoxicity. Acc. Chem. Res. 46, 743-749 (2013).

6 Johnston, A. P. R., Such, G. K., Ng, S. L. \& Caruso, F. Challenges facing colloidal delivery systems: from synthesis to the clinic. Curr. Opin. Colloid Interface Sci. 16, 171-181 (2011).

7 Martens, T. F., Remaut, K., Demeester, J., De Smedt, S. C. \& Braeckmans, K. Intracellular delivery of nanomaterials: how to catch endosomal escape in the act. Nano Today 9, 344-364 (2014).

8 Breunig, M., Lungwitz, U., Liebl, R. \& Goepferich, A. Breaking up the correlation between efficacy and toxicity for nonviral gene delivery. Proc. Natl Acad. Sci. USA 104, 14454-14459 (2007).

9 Synatschke, C. V., Schallon, A., Jérôme, V., Freitag, R. \& Müller, A. H. E. Influence of polymer architecture and molecular weight of poly(2-(dimethylamino)ethyl methacrylate) polycations on transfection efficiency and cell viability in gene delivery. Biomacromolecules 12, 4247-4255 (2011).

10 Cabral, H., Matsumoto, Y., Mizuno, K., Chen, Q., Murakami, M., Kimura, M., Terada, Y., Kano, M. R., Miyazono, K., Uesaka, M., Nishiyama, N. \& Kataoka, K. Accumulation of sub-100 nm polymeric micelles in poorly permeable tumours depends on size. Nat. Nanotechnol. 6, 815-823 (2011).

11 Jokerst, J. V., Lobovkina, T., Zare, R. N. \& Gambhir, S. S. Nanoparticle PEGylation for imaging and therapy. Nanomedicine 6, 715-728 (2011).

12 Ishihara, T., Takeda, M., Sakamoto, H., Kimoto, A., Kobayashi, C., Takasaki, N., Yuki, K., Tanaka, K., Takenaga, M., Igarashi, R., Maeda, T., Yamakawa, N., Okamoto, Y., Otsuka, M., Ishida, T., Kiwada, H., Mizushima, Y. \& Mizushima, T. Accelerated blood clearance phenomenon upon repeated injection of PEG-modified PLA-nanoparticles. Pharm. Res. 26, 2270-2279 (2009).

13 Saadati, R., Dadashzadeh, S., Abbasian, Z. \& Soleimanjahi, H. Accelerated blood clearance of PEGylated PLGA nanoparticles following repeated injections: effects of polymer dose, PEG coating, and encapsulated anticancer drug. Pharm. Res. 30, 985-995 (2013).

14 Schacher, F. H., Rupar, P. A. \& Manners, I. Functional block copolymers: nanostructured materials with emerging applications. Angew. Chem. Int. Ed. 51, 7898-7921 (2012).

15 Dang, N. M., Zhao, W.-W., Yusa, S.-I., Noguchi, H. \& Nakashima, K. Cobalt oxide hollow nanoparticles as synthesized by templating a tri-block copolymer micelle with a core-shell-corona structure: a promising anode material for lithium ion batteries. New J. Chem. 39, 4726-4730 (2015).

16 Guerlain, C., Piogé, S., Detrembleur, C., Fustin, C.-A. \& Gohy, J.-F. Self-assembly of a triblock terpolymer mediated by hydrogen-bonded complexes. J. Polym. Sci. Polym. Chem. 53, 459-467 (2015).

17 Sasidharan, M. \& Nakashima, K. Core-shell-corona polymeric micelles as a versatile template for synthesis of inorganic hollow nanospheres. Acc. Chem. Res. 47, 157-167 (2013).

18 Zhao, X. \& Liu, P. Reduction-responsive core-shell-corona micelles based on triblock copolymers: novel synthetic strategy, characterization, and application as a tumor microenvironment-responsive drug delivery system. ACS Appl. Mater. Interfaces 7, 166-174 (2014).

19 Rinkenauer, A. C., Schallon, A., Günther, U., Wagner, M., Betthausen, E., Schubert, U. S. \& Schacher, F. H. A paradigm change: efficient transfection of human leukemia cells by stimuli-responsive multicompartment micelles. ACS Nano 7, 9621-9631 (2013)

20 Barthel, M. J., Mansfeld, U., Hoeppener, S., Czaplewska, J. A., Schacher, F. H. \& Schubert, U. S. Understanding and tuning the self-assembly of polyether-based triblock terpolymers in aqueous solution. Soft Matter 9, 3509-3520 (2013).

21 Barthel, M. J., Rinkenauer, A. C., Wagner, M., Mansfeld, U., Hoeppener, S., Czaplewska, J. A., Gottschaldt, M., Träger, A., Schacher, F. H. \& Schubert, U. S. Small but powerful: co-assembly of polyether-based triblock terpolymers into sub$30 \mathrm{~nm}$ micelles and synergistic effects on cellular interactions. Biomacromolecules 15 , 2426-2439 (2014)

22 Neto, N., Dilauro, C., Castellu, E. \& Califano, S. Vibrational spectra and molecular conformation of cyclenes .I. Vibrational assignemnt and valence force field of cyclohexene and cyclohexene-D10. Spectrochim. Acta A Mol. Spectr. A23, 1763 (1967).

23 Nishimura, Y., Tsuboi, M., Sato, T. \& Aoki, K. Conformation-sensitive Raman lines of mononucleotides and their use in a structure-analysis of polynucleotides-guanine and cytosine nucleotides. J. Mol. Struct. 146, 123-153 (1986). 
24 Press, A. T., Ungelenk, L., Rinkenauer, A. C., Gröger, M., Lehmann, F., Mosig, A., Schubert, U. S., Clemens, M. G. \& Bauer, M. A new fluorescent dye for cell tracing and mitochondrial imaging in vitro and in vivo. J. Biophoton. 9, 888-900 (2015)

25 Press, A. T., Traeger, A., Pietsch, C., Mosig, A., Wagner, M., Clemens, M. G., Jbeily, N. Koch, N., Gottschaldt, M., Bézière, N., Ermolayev, V., Ntziachristos, V., Popp, J., Kessels, M. M., Qualmann, B., Schubert, U. S. \& Bauer, M. Cell type-specific delivery of short interfering RNAs by dye-functionalised theranostic nanoparticles. Nat. Commun. 5 (2014).

26 Winter, M. E. N-FINDR: an algorithm for fast autonomous spectral end-member determination in hyperspectral data. Proc. SPIE 3753, Imaging Spectrometry V 3753, 266-275 (1999).

$27 R$ Development Core Team, RR (Vienna, Austria, 2011).

28 Ryan, C. G., Clayton, E., Griffin, W. L., Sie, S. H. \& Cousens, D. R. SNIP, a statisticssensitive background treatment for the quantitative analysis of PIXE spectra in geoscience applications. Nucl. Instr. Meth. Phys. Res. B 34, 396-402 (1988).

29 Barthel, M. J., Babiuch, K., Rudolph, T., Vitz, J., Hoepnner, S., Gottschaldt, M., Hager, M. D., Schacher, F. H., Schubert, U. S. Bis-hydrophilic and functional triblock terpolymers based on polyethers: synthesis and self-assembly in solution. J. Polym. Sci. A Polym. Chem. 50, 2914-2923 (2012).

30 Halacheva, S., Rangelov, S. \& Tsvetanov, C. Poly(glycidol)-based analogues to pluronic block copolymers. synthesis and aqueous solution properties. Macromolecules 39 , 6845-6852 (2006).

31 Ding, H. M., Tian, W. D. \& Ma, Y. Q. Designing nanoparticle translocation through membranes by computer simulations. ACS Nano 6, 1230-1238 (2012).

32 Ting, C. L. \& Wang, Z.-G. Minimum free energy paths for a nanoparticle crossing the lipid membrane. Soft Matter 8, 12066-12071 (2012).

33 Leroueil, P. R., Hong, S., Mecke, A., Baker, J. R., Orr, B. G. \& Banaszak Holl, M. M. Nanoparticle interaction with biological membranes: does nanotechnology present janus face? Acc. Chem. Res. 40, 335-342 (2007).
34 Betthausen, E., Drechsler, M., Förtsch, M., Schacher, F. H. \& Müller, A. H. E. Dua stimuli-responsive multicompartment micelles from triblock terpolymers with tunable hydrophilicity. Soft Matter 7, 8880-8891 (2011).

35 Perry, S. W., Norman, J. P., Barbieri, J., Brown, E. B. \& Gelbard, H. A. Mitochondrial membrane potential probes and the proton gradient: a practical usage guide. BioTechniques 50, 98-115 (2011).

36 Liu, T., Li, L., Fu, C., Liu, H., Chen, D. \& Tang, F. Pathological mechanisms of liver injury caused by continuous intraperitoneal injection of silica nanoparticles. Biomaterials 33, 2399-2407 (2012).

37 Moghimi, S. M., Hunter, A. C. \& Andresen, T. L. Factors controlling nanoparticle pharmacokinetics: an integrated analysis and perspective. Annu. Rev. Pharmacol. Toxicol. 52, 481-503 (2012).

38 Owens, D. E. \& Peppas, N. A. Opsonization, biodistribution, and pharmacokinetics of polymeric nanoparticles. Int. J. Pharm. 307, 93-102 (2006).

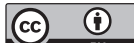

This work is licensed under a Creative Commons Attribution 4.0 International License. The images or other third party material in this article are included in the article's Creative Commons license, unless indicated otherwise in the credit line; if the material is not included under the Creative Commons license, users will need to obtain permission from the license holder to reproduce the material. To view a copy of this license, visit http:// creativecommons.org/licenses/by/4.0/

(C) The Author(s) 2017

Supplementary Information accompanies the paper on the NPG Asia Materials website (http://www.nature.com/am) 